\title{
TRATAMENTO DA EPIFISIÓLISE PROXIMAL FEMORAL GRAVE COM OSTEOTOMIA SUBTROCANTÉRICA PELO MÉTODO DE ILIZAROV
}

\author{
TREATMENT OF PROXIMAL FEMORAL EPIPHYSIOLYSIS WITH \\ SUBTROCHANTERIC OSTEOTOMY BY THE ILIZAROV METHOD
}

Leandro de Freitas Spinelli ${ }^{1}$, Samuel Faccioni ${ }^{2}$, Jung Ho Kim³ ${ }^{3}$, Luis Gustavo Calieron ${ }^{4}$, Juan Carlo Mendieta Rojas ${ }^{5}$

\section{RESUMO}

Objetivos: Avaliar o tratamento da epifisiólise femoral proximal grave através da osteotomia subtrocantérica pelo método de Ilizarov e propor uma metodologia de cálculo para estimar a correção necessária. Métodos: Foi realizado um estudo de casos de pacientes acometidos por epifisiólise do fêmur proximal grave, submetidos a osteotomia subtrocantérica com fixação externa multiplanar, avaliados no pós-operatório pela classificação modificada de Southwick. Foi desenvolvida uma metodologia de cálculo para estimar a correção angular necessária no ato cirúrgico. Resultados: Foram avaliados 13 quadris, quatro à esquerda (30,8\%) e nove à direita $(69,2 \%)$, de maioria $(61,5 \%)$ do sexo masculino, com idade média de 14 anos. Obtiveramse como parâmetros para dor e função $30,8 \%$ de excelentes resultados, 46,2\% de bons e 23,0\% de regulares, não sendo observados resultados ruins. $\mathrm{O}$ arco de movimento apresentou $46,2 \%$ de excelentes resultados, $30,8 \%$ de bons e $23,0 \%$ de regulares. Radiologicamente, foram obtidos $7,7 \%$ de excelentes resultados, $69,2 \%$ de bons, $15,4 \%$ de regulares e $7,7 \%$ de ruins. Conclusões: O tratamento percutâneo da epifisiólise proximal grave pelo método de Ilizarov como opção terapêutica apresenta bons índices de melhora da dor e função. O método de cálculo proposto auxilia de forma positiva no planejamento cirúrgico, apresentando variações entre os ângulos medidos e calculados da ordem de $3^{\circ}$.

Descritores - Epífise deslocada; Osteotomia; Fixadores externos; Técnica de Ilizarov

\section{ABSTRACT}

Objectives: This paper evaluates the treatment of severe proximal femoral epiphysiolysis through subtrochanteric osteotomy by the Ilizarov method and propose a new method for calculating the necessary correction. Methods: a case study was carried out with patients affected by severe proximal femoral epiphysiolysis, submitted to subtrochanteric osteotomy with external multiplanar fixation, and evaluated postoperatively by the modified Southwick classification. A method was developed for calculating the necessary angular correction at surgery procedure. Results: thirteen hips were analysed, four on the left (30.8\%) and nine to the right (69.2\%). The majority of patients (61.5\%) were male, with a mean age of 14 yrs. In relation to pain and function, results were excellent: $30.8 \%$; good: $46.2 \%$; and regular: $23.0 \%$. There were no poor results in relation to these two aspects. The results for mobility were: excellent 46.2\%; good 30.8\%; and regular: $23.0 \%$. Radiographically, the results were: excellent $7.7 \%$; good: $69.2 \%$; regular: $15.4 \%$; and poor: 7.7\%. Conclusions: percutaneous treatment of the severe proximal epiphysiolysis by the Ilizarov method is a good treatment option with good indices for improvement of pain and function. The proposed calculation method can help in planning surgery procedures, showing small variations between preoperative calculated angles and measured postoperative angles.

Keywords - Dislocated epiphyses; Osteotomy; External fixators; Ilizarov technique

\footnotetext{
1 - Médico Ortopedista e Traumatologista do Instituto de Ortopedia e Traumatologia de Passo Fundo/RS e Hospital de Clínicas de Porto Alegre/UFRGS.

2 - Médico Ortopedista e Traumatologista/Especialista em Cirurgia do Quadril do Instituto de Ortopedia e Traumatologia de Passo Fundo/RS.

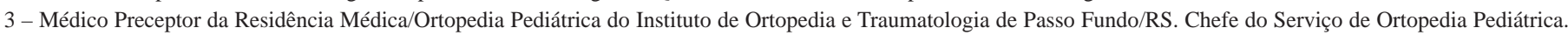

4 - Médico Preceptor da Residência Médica/Ortopedia Pediátrica do Instituto de Ortopedia e Traumatologia de Passo Fundo/RS

5 - Médico Especialista em Ortopedia Pediátrica do Instituto de Ortopedia e Traumatologia de Passo Fundo/RS.
}

Trabalho realizado no Instituto de Ortopedia e Traumatologia de Passo Fundo, RS.

Correspondência: IOT - Rua Uruguai, 2.050 - Centro.- 99010-150 - Passo Fundo. RS - Brasil. E-mail: iot@iotrs.com.br 


\section{INTRODUÇÃO}

A epifisiólise proximal femoral ocorre devido a uma debilidade mecânica da fise de crescimento, causando um deslocamento anterossuperior da metáfise sobre a epífise femoral, produzindo uma deformidade tridimensional, com a metáfise posicionada em varo, extensão e rotação externa, porém mantendo a relação do acetábulo com a cabeça femoral ${ }^{(1,2)}$.

O tratamento do escorregamento da epífise femoral proximal (EEPF) consiste primariamente na prevenção da progressão do escorregamento, evitando assim maior deformidade e complicações com a sua progressão, enquanto ocorre o remodelamento do colo femoral.

O tratamento tardio está reservado aos casos em que a deformidade em retroversão do colo femoral causa sintomas (dor, diminuição do arco de movimento, dificuldades de marcha), estando relacionada com o impacto femoro-acetabular anterior, acarretando o desenvolvimento precoce de osteoartrite ${ }^{(3)}$.

As osteotomias do fêmur proximal procuram corrigir as deformidades que ocorrem na epífise de crescimento e, embora pareça lógica a correção da deformidade no próprio local, as osteotomias corretivas nessa região geralmente estão associadas a altos índices de complicações, especialmente a necrose avascular.

A osteotomia subtrocantérica triplanar de Southwick é uma boa opção para a correção da deformidade do colo porque evita essa região, comumente associada a complicações, sendo realizada em nível inferior, provocando uma deformidade compensatória em flexão, valgo e rotação interna no nível subtrocantérico.

O presente trabalho avaliou 13 pacientes com escorregamento proximal do fêmur e propõe uma metodologia de cálculo para estimar a correção necessária no ato cirúrgico, considerando-se um ângulo crítico de correção a partir do qual não haveria mais contato direto das corticais.

\section{MÉTODOS}

O presente trabalho abrange um estudo de casos e foi desenvolvido no IOT - Instituto de Ortopedia e Traumatologia de Passo Fundo/RS. Os pacientes foram avaliados retrospectivamente de 2000 a 2007, correspondendo a 13 quadris. Foram incluídos neste trabalho pacientes acometidos por epifisiólise do fêmur proximal classificados como graus II e III de Southwick.

Todos os pacientes foram submetidos a uma completa anamnese e exame físico e exames complementares de radiografia de pelve em anteroposterior e Lauenstein. Foi realizada a medida do ângulo entre a epífise e a diáfise, na radiografia em abdução e rotação externa, sendo consideradas normais medidas de até $12^{\circ}$, escorregamentos leves $\left(<30^{\circ}\right)$, moderados $\left(30-50^{\circ}\right)$ e severas $\left(>50^{\circ}\right)$ com sinal de Drennan positivo. Após o procedimento cirúrgico, os pacientes foram avaliados através da classificação modificada de Southwick ${ }^{(4)}$, que consiste na avaliação clínica e radiográfica, conforme Tabela 1 .

Os pacientes selecionados foram submetidos à osteotomia subtrocantérica com fixação externa multiplanar, associada à fixação in situ, dependendo da presença da fise aberta. Como rotina no segmento pósoperatório, os pacientes são orientados a não apoiar o lado operado e iniciam um programa de fisioterapia para arco de movimento imediatamente no pós-operatório. Os pacientes com fixação in situ contralateral realizada no mesmo procedimento (casos bilaterais) não deambulam. Após orientações e alta hospitalar, os pacientes retornam ao ambulatório em uma semana para revisão e curativos e, em duas e quatro semanas,

Tabela 1 - Classificação de Southwick modificada(4)

\begin{tabular}{|c|c|c|c|c|}
\hline & Excelente & Bom & Regular & Ruim \\
\hline Dor & Ausente & Presente com exercícios & $\begin{array}{l}\text { Usualmente com exercícios e } \\
\text { ocasionalmente com atividade normal }\end{array}$ & Constante \\
\hline Função & Sem limitação & $\begin{array}{l}\text { Cansaço ocasional, pode caminhar mais que } \\
\text { uma milha }\end{array}$ & $\begin{array}{l}\text { Cansa-se facilmente, caminha até um quarto } \\
\text { de milha }\end{array}$ & $\begin{array}{l}\text { Não pode caminhar um quarto } \\
\text { de milha }\end{array}$ \\
\hline Arco de movimento & até $20^{\circ}$ perda lado normal & $40^{\circ}$ perda lado normal & $60^{\circ}$ perda lado normal & Maior que $60^{\circ}$ \\
\hline Radiologia & $\begin{array}{l}\text { Sem incongruência ou } \\
\text { estreitamento }\end{array}$ & $\begin{array}{l}\text { Nenhuma Incongruência, moderado } \\
\text { estreitamento ou perda da esfericidade, sem } \\
\text { cistos ou alterações degenerativas }\end{array}$ & $\begin{array}{l}\text { Moderada incongruência, moderadas } \\
\text { alterações artríticas, sem necrose avascular }\end{array}$ & $\begin{array}{l}\text { Incongruência e degeneração } \\
\text { severa, necrose avascular }\end{array}$ \\
\hline
\end{tabular}


para radiografias de controle, mantendo acompanhamento radiográfico a cada 30 dias até a consolidação e fisioterapia continuamente.

A partir de radiografias obtidas na fase inicial da pesquisa, foi desenvolvida uma metodologia de cálculo que pudesse estimar a correção necessária no ato operatório, que consiste em uma análise das radiografias em anteroposterior e Lauenstein (Figuras 1A e 1B), de forma semelhante à proposta por Southwick. Basicamente, o cálculo compara o lado normal com o lado comprometido. Inicialmente, foram traçadas as linhas cervicodiafisárias nos lados normal e acometido, seguindo-se das medidas dos respectivos ângulos (Figuras 1C e 1D). A seguir, foram traçadas novas linhas dos centros das cabeças femorais ao ângulo de intersecção cervicodiafisários, definindo os ângulos $\alpha$ e $\beta$ conforme Figuras 1E e 1F. Uma linha perpendicular ao eixo diafisário passando imediatamente abaixo do pequeno trocânter foi traçada (na radiografia anteroposterior e no Lauenstein), formando um triângulo equilátero de ângulo menor $(\gamma$ e $\omega)$ na intersecção das linhas cervicodiafisárias (Figuras $1 \mathrm{G}$ e $1 \mathrm{H}$ ). Os ângulos $\alpha$ e $\gamma$ e os ângulos $\beta$ e $\omega$ são sobrepostos (Figuras $1 \mathrm{I}, 1 \mathrm{~J}, 1 \mathrm{~K}, 1 \mathrm{~L}$, $1 \mathrm{M}$ e $1 \mathrm{~N}$ ) e o planejamento cirúrgico pode ser efetuado, observando-se:

$\alpha<\gamma$ : pode-se efetuar a osteotomia, obtendo-se contato entre as corticais;

$\alpha=\gamma$ : ângulo crítico limite de contato das corticais na radiografia em anteroposterior;

$\alpha>\gamma$ : não há contato das corticais na radiografia em anteroposterior;

$\beta<\omega$ : pode-se efetuar a osteotomia, obtendo-se contato entre as corticais;

$\beta=\omega$ : ângulo crítico limite de contato das corticais na radiografia em Lauenstein;

$\beta>\omega$ : não há contato entre as corticais na radiografia em Lauenstein.

Define-se, portanto, um ângulo crítico de correção, no qual, a partir deste, não se obtém mais qualquer contato cortical, inviabilizando o uso da técnica da osteotomia em três planos pelo método de Ilizarov. Vale salientar que o osso apresenta forma praticamente elíptica nessa região e que o ângulo crítico final de ajuste seria uma resultante tridimensional entre a intersecção dos planos radiográficos, mas o cálculo puramente bidimensional nas radiografias em anteroposterior e Lauenstein já pode fornecer uma excelente aproximação.
A metodologia de cálculo descrita foi aplicada no pré-operatório e avaliada a correção cirúrgica no pósoperatório para todos os pacientes. O procedimento cirúrgico foi indicado após a verificação da presença de contato das corticais, estimado pelo cálculo proposto.

A análise dos dados foi realizada de forma descritiva através do uso do programa SPSS for Windows v.15 (SPSS Inc., Chicago, Illinois, EUA).

\section{TÉCNICA OPERATÓRIA}

O paciente é posicionado em decúbito dorsal sob anestesia geral. É realizada, inicialmente, a fixação in situ da fise proximal do fêmur dos pacientes com potencial progressão do deslizamento pela presença de fise ainda aberta com um parafuso canulado (Figuras 2A e 2B). Então, sob fluoroscopia, inserem-se os pinos de Schanz proximais paralelamente ao plano horizontal e o semianel de Ilizarov (em adução para avaliar a congruência articular, projetando-se a valgização necessária) (Figuras 2C e 2D). Após, com a rótula centrada, são inseridos os pinos de Schanz distais, também paralelos à mesa cirúrgica. Como resultado, observa-se que o semianel superior posiciona-se em flexão com os pinos proximais orientados cranialmente em relação ao semianel inferior (Figura 2E). Realiza-se a osteotomia imediatamente abaixo do pequeno trocânter (Figura 2F). Corrige-se a deformidade alinhando-se paralelamente os dois semianéis e fixando-os com barras conectoras (Figura 2G), sendo possível também corrigir deformidades residuais pela suas conexões. As Figuras 2H e 2I apresentam o aspecto radiográfico final na fluoroscopia em anteroposterior e perfil.

\section{RESULTADOS}

Entre os pacientes selecionados para o estudo, oito $(61,5 \%)$ eram do sexo masculino e cinco $(38,5 \%)$ do feminino, sendo dez brancos $(76,9 \%)$ e três afro-descendentes $(23,1 \%)$. Foram operados quatro quadris esquerdos e nove direitos. A idade mínima foi de 11 anos e seis meses e máxima de 17 anos e cinco meses (média de 14 anos e cinco meses), com seguimento médio de dois anos.

Quatro pacientes apresentavam fechamento fisário, detectado através de radiografias simples, não sendo realizada fixação in situ. Quatro pacientes apresentaram a doença bilateral, mas nesses casos, em um dos lados acometidos havia escorregamento até o grau I e foi realizada fixação in situ da fise do quadril contralateral ao da osteotomia. 


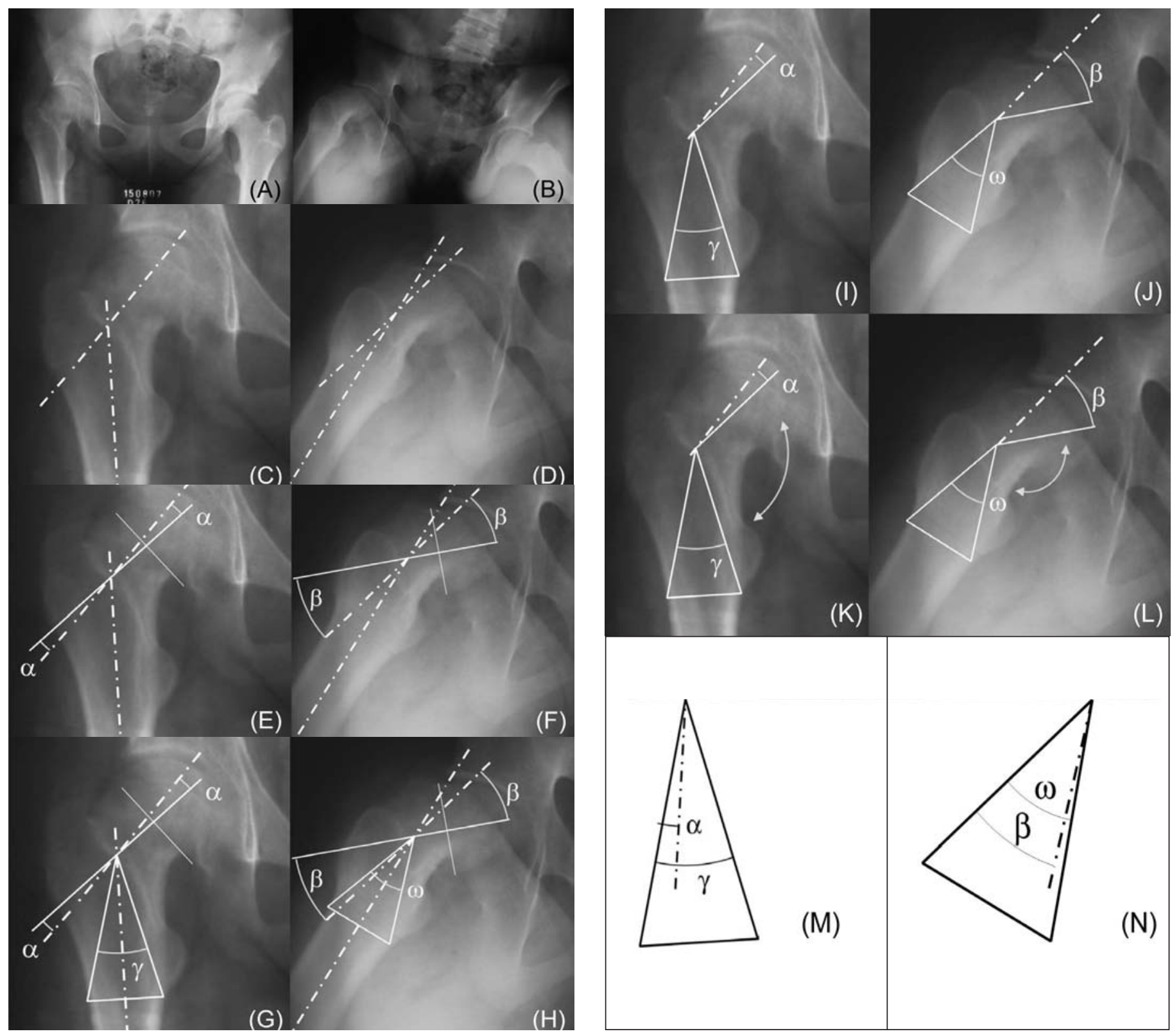

Figura 1 - Método para o cálculo da estimativa do ângulo crítico: radiografias em anteroposterior (A) e perfil (B), mostrando o escorregamento epifisário femoral proximal à direita; $(C)$ e (D) definição dos ângulos cervicodiafisários nas incidências anteroposterior e Lauenstein; (E) e (F) medidas dos ângulos de correção $\alpha$ e $\beta$; $(G)$ e $(H)$ determinação dos ângulos críticos $\gamma$ e $\omega$; (I), (J), (K), (L), (M) e $(\mathrm{N})$ isolamento dos ângulos críticos no anteroposterior e Lauenstein e comparação com os ângulos necessários para correção

Nove pacientes apresentavam escorregamento epifisário crônico (tempo de sintomatologia maior que seis semanas) e quatro, escorregamento crônico agudizado.

O tempo médio do procedimento desde a indução da anestesia geral foi de 90 minutos e o tempo médio de permanência com o fixador externo foi de 3,5 meses (dois a sete meses). Nenhum paciente necessitou reposição sanguínea no trans ou no pós-operatório ou apresentou instabilidade hemodinâmica. $\mathrm{O}$ fato se deve à cirurgia ser percutânea, inserindo-se os pinos de Schanz sob orientação fluoroscópica e fazendo-se uma mini-incisão para a osteotomia em local de zona de segurança.
Como complicações encontradas nos procedimentos, observou-se infecção no trajeto dos pinos em três pacientes e em nenhum destes foi necessária antibioticoterapia endovenosa, já que não houve casos de infecção profunda. Uma desvantagem potencial do método seria a artrofibrose por dor causada pelo próprio fixador externo, mas essa complicação não foi encontrada, já que houve fisioterapia imediata no pós-operatório.

Na avaliação clínica de Southwick ${ }^{(4)}$ da dor e função, obtivemos quatro excelentes (30,8\%), seis bons (46,2\%) e três resultados regulares (23,0\%), não sendo observados resultados ruins nesses requisitos. $\mathrm{O}$ arco de movi- 


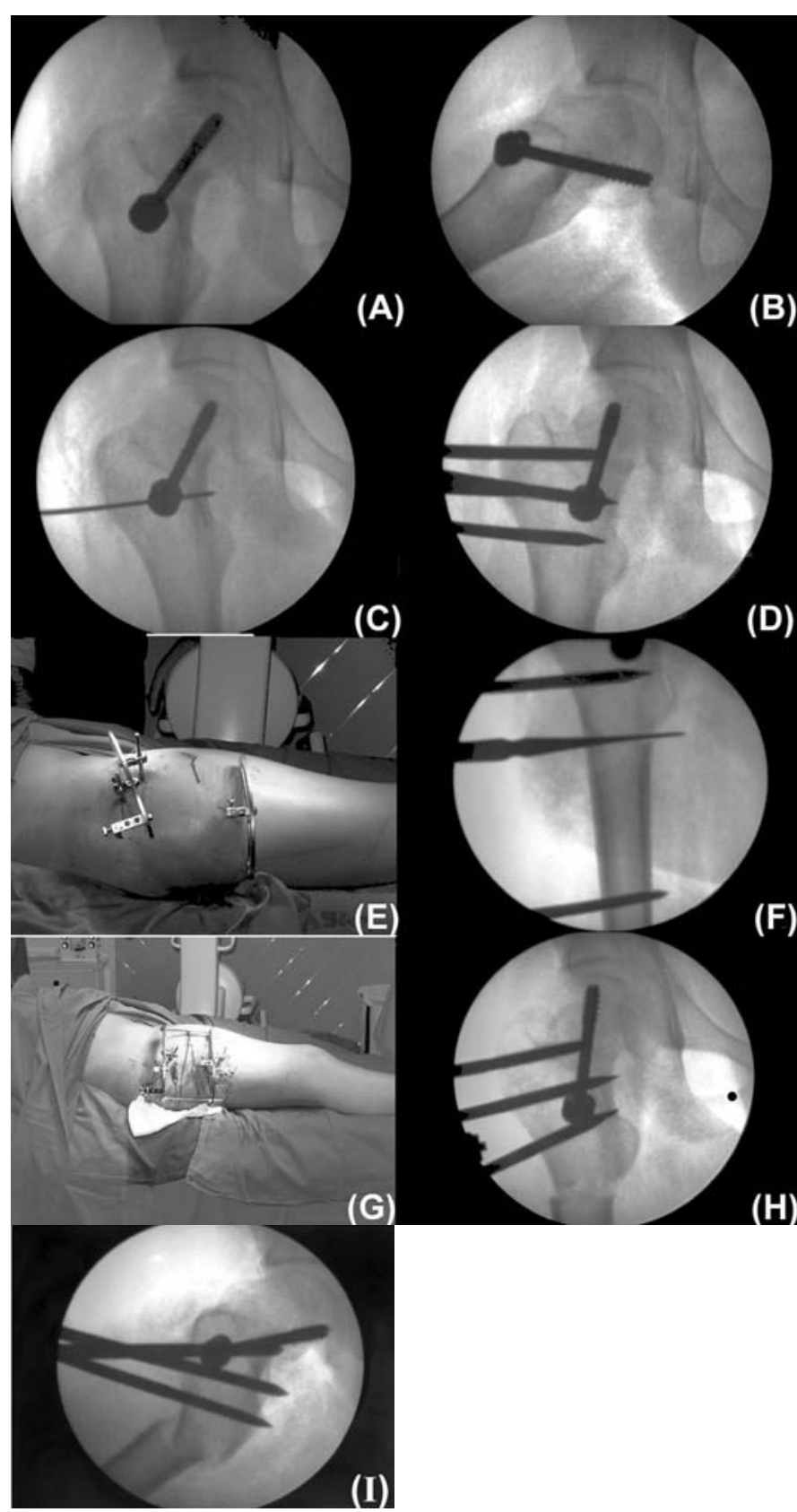

Figura 2 - Técnica operatória

mento apresentou seis excelentes resultados (46,2\%), quatro bons (30,8\%) e três regulares (23,0\%). Nos casos em que foram detectadas alterações bilaterais, apesar de fixados bilateralmente, destacamos que o lado contralateral apresentava escorregamentos até o grau I, não havendo alteração significativa do arco de movimento. Radiograficamente, foram observados nove resultados bons (69,2\%), um excelente (7,7\%), dois regulares (15,4\%) e um ruim (7,7\%). A eficácia do tratamento demonstra que a técnica percutânea pode ser bem indicada no tratamento do escorregamento femoral proximal. Os resultados regulares da avaliação radiológica estiveram associados à hipocorreção do varo, que necessitou epifisiodese do grande trocânter em um caso e, no outro, de distalização do grande trocânter em função de claudicação do paciente. A única avaliação radiológica ruim foi associada ao desenvolvimento de condrólise.

Quanto ao método de cálculo proposto para estimar as correções, observou-se que em todos os casos avaliados o planejamento pré-operatório mostrava ângulos abaixo do crítico, fato que previa que a osteotomia poderia ser realizada sem que houvesse perda de contato entre as corticais. Nessa parte do estudo, observamos a importância de uma radiografia de qualidade, que pode determinar variações nas angulações medidas quando comparamos exames sem definição ou com mau posicionamento do paciente, que pode conferir rotações inapropriadas. O método mostrou-se adequado para estimar a correção necessária, com ângulos medidos no pré-operatório e a correção obtida em pós-operatório com diferenças médias de $3^{\circ}$.

\section{DISCUSSÃO}

O principal objetivo de corrigir as deformidades moderadas e severas no deslizamento epifisário proximal do fêmur é melhorar a função da marcha e a mobilidade do quadril e, secundariamente, a deformidade pré-artrósica, restaurando a anatomia e a biomecânica normal do quadril e, teoricamente, evitando a coxartrose secundária por longo prazo. A correção pode ser obtida de várias maneiras ${ }^{(3)}$, sendo utilizadas as osteotomias como ferramentas de rotina para o tratamento de diversas patologias ortopédicas do quadril em desenvolvimento (displasias, doença de Legg-Perthes-Calvè, coxa vara e escorregamento epifisário do fêmur proximal).

Dentre os métodos disponíveis para o tratamento para EEFP, a fixação in situ permanece o procedimento de escolha no manejo inicial de EEFP estáveis, ocorrendo baixa incidência de progressão do escorregamento, osteonecrose e condrólise ${ }^{(1)}$.

Após o tratamento inicial, a deformidade em rotação externa gradualmente melhora conforme a inflamação diminui e a deformidade se remodela ${ }^{(1)}$, porém, clinicamente, Aronson e Carlson ${ }^{(5)}$, em uma série de 80 quadris fixados in situ, observaram, após acompanhamento médio de 3,3 anos, 70\% de bons e excelentes resultados. Entretanto, quando os autores avaliaram os quadris pelo grau de deslizamento, obtiveram $86 \%$ de bons resultados nos casos leves, 55\% nos casos moderados e apenas $27 \%$ nos casos severos, o que permite concluir que o 
grau de deslizamento está diretamente relacionado com o prognóstico do paciente, sendo selecionados para o procedimento de fixação in situ apenas pacientes com EEFP leve a moderado.

O tratamento de casos moderados e severos (com escorregamentos maiores que $30^{\circ}$ ) permanece um desafio ao ortopedista em vista das complicações inerentes à própria doença do paciente e às alternativas cirúrgicas para esses casos. O uso de osteotomias há muito tem sido estudado no manejo do EEFP. Alguns autores propõem a correção da região cervical intracapsular e defendem que essa teoricamente seria a única opção capaz de corrigir a deformidade completamente e não prejudicar uma cirurgia reconstrutiva no futuro ${ }^{(6)}$; entretanto, essas são cirurgias associadas mais comumente a complicações. Dunn e Angel ${ }^{(7)}$ e Biring et $a l^{(8)}$, em sua série de pacientes tratados com osteotomia cuneiforme intracapsular, obtiveram altas taxas de necrose avascular e condrólise, chegando a 39\% e 17\%, respectivamente, em pacientes com escorregamento crônico agudizado. $\mathrm{O}$ risco de necrose avascular é considerável e Gage et al ${ }^{(9)}$ demonstraram 38\% de prevalência de necrose em 77 quadris tratados com osteotomia do colo femoral, sendo que não relacionaram a severidade do deslocamento ou a correção obtida. Diab et al ${ }^{(10)}$ encontraram baixas taxas de complicações, porém, a osteotomia subcapital foi associada mais comumente à reoperação. A realização de osteotomias subtrocantéricas pela via de acesso habitual, descrita por Southwick ${ }^{(4)}$, além da exposição cirúrgica em excesso, também implica maior perda sanguínea para remoção do fragmento trapezoidal e aumenta o risco de encurtamento do membro ${ }^{(9)}$, podendo ainda haver a necessidade de imobilização com órteses, dependendo da estabilidade cirúrgica obtida. Além disso, pode ser necessário novo procedimento cirúrgico aberto para remoção do material de síntese no futuro. Schai et $a l^{(6)}$, em uma série de 51 quadris tratados com osteotomia intertrocantérica, avaliados após 24 anos em média, descreveram que $55 \%$ dos quadris apresentavam-se sem alterações degenerativas e 28\%, sinais degenerativos apenas moderados. Kartenbender et al $^{(11)}$, em seu estudo de 35 pacientes e 39 quadris, observaram que após as osteotomia intertrocantéricas, com seguimento médio de 23,4 anos, eram obtidos $77 \%$ de resultados clínicos excelentes e bons.

A indicação de fixação e osteotomia subtrocantérica tem por objetivo primário estabilizar o escorregamento em associação com a correção da deformidade em um nível distante do centro da deformidade, gerando assim uma deformidade compensatória. Há controvérsias quanto à necessidade ou não da fixação da epífise femoral em conjunto com a osteotomia subtrocantérica, já que Southwick ${ }^{(4)}$ em seu artigo original pressupõe que ocorre uma fusão precoce da fise ocasionada por uma horizontalização em função da osteotomia, o que evita complicações do uso de fixação in situ, com até 10\% de necrose em algumas séries. Porém, a fixação in situ dispensa o uso de órteses pós-operatórias para imobilizar o quadril; por essa razão, no presente estudo optamos pela fixação in situ nos pacientes que se apresentavam com a fise ainda aberta.

O uso da técnica percutânea demonstra vários benefícios, dentre eles, especialmente a diminuição da perda sanguínea e menor morbidade cirúrgica, além de evitar problemas associados à fixação interna com placas, como a possibilidade de acometer a fise de crescimento e a necessidade de segundo procedimento para remover o material de síntese e a potencial diminuição das taxas de infecção profunda ${ }^{(12)}$, além de possibilitar ajustes pós-operatórios de deformidades residuais ${ }^{(13)}$. Não houve casos de falha de implante nessa pesquisa. Beauchesne et al ${ }^{(14)}$ apresentaram 5\% de fratura após fixação com placa AO. Há indicação também nos casos em que não haja condição adequada de pele porém, há relação com taxas significativas de infecção no trajeto dos pinos, com índices na literatura que variam de 0 a $100 \%$, com média de 4 a $12 \%{ }^{(15)}$. Kishan et al ${ }^{(16)} \mathrm{em}$ seu trabalho associam a redução nas taxas de osteólise com uso de pinos revestidos por hidroxiapatita, usando técnica de inserção dos pinos sem provocar necrose térmica, com antibióticos orais precocemente. No presente trabalho utilizamos pinos de aço inox, sem obter taxas elevadas de infecção.

Um potencial risco da cirurgia percutânea é o tempo de exposição radioativa pelo uso de intensificador de imagens, para o qual não encontramos na literatura dados objetivos. Quanto ao tempo médio do procedimento desde a indução da anestesia, obtivemos média de 90 minutos, com tempo médio de permanência com o fixador externo de 3,5 meses (dois a sete meses). Colyer ${ }^{(17)}$ associou o uso por um período de tempo semelhante (três a quatro meses). Não encontramos na literatura referências aos tempos cirúrgicos.

El-Mowafi et al ${ }^{(18)}$ não encontraram diferenças significativas na avaliação pós-operatória de pacientes submetidos à osteotomia de Southwick versus os- 
teotomia do colo femoral, obtendo bons e excelentes resultados em $90 \%$ e $86,7 \%$ dos pacientes, respectivamente, porém, citam como vantagens da osteotomia do colo femoral a capacidade de preservar a anatomia do fêmur proximal. Complicações como condrólise foram mais comuns após osteotomias subtrocantéricas e a necrose avascular após osteotomias do colo femoral. Diab et $a l^{(19)}$, comparando fixação in situ isolada com osteotomia intertrocantérica associada com fixação in situ, não encontraram diferença significativa na função do quadril, porém, obtendo sucesso na melhora da mobilidade com a osteotomia.

Quanto à definição do ângulo crítico, vale salientar que este pode ser influenciado pelas seguintes variáveis: a) qualidade da radiografia, que pode mascarar a identificação das linhas de contorno no anteroposterior e perfil, podendo alterar os ângulos medidos; b) técnica cirúrgica e correção realizada no procedimento cirúrgico, no qual o cirurgião não necessariamente corrige com exatidão o ângulo previamente medido. No presente estudo, a diferença entre os ângulos medidos e corrigidos ficou em aproximadamente $3^{\circ}$, tanto na radiografia em anteroposterior como no Louenstein. Consideramos que as definições dos ângulos pré e pós-operatórios pode servir como guia para futuros procedimentos.

\section{REFERÊNCIAS}

1. Aronsson DD, Loder TR, Breuer JG, Weinstein LS. Slipped capital femoral epiphysis: current concepts. J Am Acad Orthop Surg. 2006;14(12):666-79.

2. Rab GT. The geometry of slipped capital femoral epiphysis: implications for movement, impingement and corrective osteotomy. J Pediatr Orthop. 1999;19(4):419-24.

3. Cabral FP, Freitas E, Penedo JL, Rondineli P, Carvalho PI, Chaparro JC. Osteotomia tridimencional no tratamento do escorregamento epifisário superior do fêmur. Rev Bras Ortop. 1997;32(10):797-800.

4. Southwick WO. Osteotomy through the lesser trochanter for slipped capital femoral epiphysis. J Bone Joint Surg Am. 1967;49(5):807-35

5. Aronson DD, Carlson WE. Slipped capital femoral epiphysis: a prospective study of fixation with single screw. J Bone Joint Surg Am. 1992;74(8):810-9.

6. Schai PA, Exner GU, Hänsch O. Prevention of secondary coxartrhosis in slipped capital femoral epiphisys, a long-term follow-up study after corretive intertrochanteric osteotomy. J Pediatr Orthop. 1996;5(3):135-43.

7. Dunn DM, Angel JC. Replacement of the femoral head by open operation in severe adolescent slipping of the upper femoral epiphysis. J Bone Joint Surg Br. 1978;60(3):394-403.

8. Biring GS, Hashemi-Najad A, Catterall A. Outcomes of subcapital cuneiform osteotomy for the treatment of severe slipped capital femoral epiphysis after skeletal maturity. J Bone Joint Surg Br. 2006;88(10):1379-84.

9. Gage JR, Sundberg AB, Nolan DR, Sletten RG, Winter RB. Complications after cuneiform osteotomy for moderately or severely slipped capital femoral epiphysis. J. Bone Joint Surg Am. 1978;60(2):157-65

10. Diab M, Hresko MT, Millis MB. Intertrochanteric versus subcapital osteotomy in slipped capital femoral epiphisys. Clin Orthop Relat Res. 2004;(427):204-12.
Nos casos em que havia acometimento bilateral, nenhum desses apresentou escorregamento maior que grau I em um dos lados acometidos e, dessa forma, um dos lados foi apenas fixado in situ. O cálculo, a princípio, se baseia no traçado das linhas no lado normal e no comprometido, de forma comparativa. Quando houver escorregamento bilateral graus II ou III, sugerimos que a correção dos ângulos de inclinação e declinação seja considerada pelas médias normais encontradas na literatura, $135^{\circ}-145^{\circ}$ de ângulo de inclinação e até $15^{\circ}$ de declinação.

Salientamos que, como os casos são severos, não se restabelece uma anatomia normal, uma vez que a osteotomia é realizada fora do centro de CORA. Entretanto, o procedimento cirúrgico é rápido, minimamente invasivo, restabelece a marcha normal evitando Trendelemburg e corrige o comprimento do membro.

\section{CONCLUSÕES}

Os resultados obtidos demonstram que a técnica percutânea é bem indicada no tratamento do escorregamento femoral proximal, com os pacientes apresentando melhora da dor e função. Quanto ao método de cálculo proposto para estimar as correções, este mostrou ser uma importante ferramenta para planejar o procedimento e estimar a correção necessária.
11. Kartenbender K, Cordier W, Katthagen B. Long-term follow-up study after corrective Imhäuser osteotomy for severe slipped capital femoral epiphysis. J Pediatr Orthop. 2000;20(6):749-56.

12. Sabharwal S, Mittal R, Cox G. Percutaneous triplanar femoral osteotomy correction for developmental coxa vara: a new technique. J Pediatr Orthop. 2005;25(1):28-33.

13. Ito $H$, Minami A, Suzuki $K$, Matsuno T. Three-dimensionally corrective external fixator system for proximal femoral osteotomy. J Pediatr Orthop. 2001;21(5):652-6

14. Beauchesne R, Miller F, Moseley C. Proximal femoral osteotomy using the AO fixed-angle blade plate. J Pediatr Orthop. 1992;12(6):735:40.

15. Giordano V, Knackfuss IG, Caldas C, Giordano M, Gomes RC, Kussmann G, et al. Infecção no trajeto dos fios e pinos do fixador externo de llizarov: estudo bacteriológico. Rev Bras Ortop. 2000;35(1):29-34.

16. Kishan S, Sabharwal S, Behrens F, Reilly M, Sirkin M. External fixation of the femur: basic concepts. Tech Orthop. 2002;17(2):239-44.

17. Colyer RA. Compression external fixation after biplane femoral trochanteric osteotomy for severe slipped capital femoral epiphysis. J Bone Joint Surg Am. 1980;62(4):557-60.

18. El-Mowafi H, El-Adl G, El-Lakkany MR. Extracapsular base of neck osteotomy versus Southwick osteotomy in treatment of moderate to severe chronic slipped capital femoral epiphysis. J Pediatr Orthop. 2005;25(2):171-7.

19. Diab AM, Daluvoy BS, Snyder BD, Kasser RBJ. Osteotomy does not improve early outcome after slipped capital femoral epiphysis. J Pediatr Orthop. 2006;15(2):87-92. 